\title{
Tunable coupling engineering between superconducting resonators: From sidebands to effective gauge fields
}

\author{
Borja Peropadre, ${ }^{1}$ David Zueco, ${ }^{2,3}$ Friedrich Wulschner, ${ }^{4}$ Frank Deppe, ${ }^{4,5}$ Achim Marx, ${ }^{4}$ \\ Rudolf Gross, ${ }^{4,5}$ and Juan José García-Ripoll ${ }^{1}$ \\ ${ }^{1}$ Instituto de Física Fundamental, IFF-CSIC, Calle Serrano 113b, E-28006 Madrid, Spain \\ ${ }^{2}$ Instituto de Ciencia de Materiales de Aragón y Departamento de Física de la Materia Condensada, CSIC-Universidad de Zaragoza, \\ Calle de Pedro Cerbuna 12, E-50009 Zaragoza, Spain \\ ${ }^{3}$ Fundación ARAID, Paseo María Agustín 36, E-50004 Zaragoza, Spain \\ ${ }^{4}$ Walther-Meißner-Institut, Bayerische Akademie der Wissenschaften, D-85748 Garching, Germany \\ ${ }^{5}$ Physik Department, Technische Universität München, James-Franck-Straße, D-85748 Garching, Germany
}

(Received 6 November 2012; revised manuscript received 19 February 2013; published 5 April 2013)

\begin{abstract}
In this work we show that a tunable coupling between microwave resonators can be engineered by means of simple Josephson junctions circuits, such as dc and rf superconducting quantum interference devices. We show that by controlling the time dependence of the coupling it is possible to switch on and off and modulate the cross-talk and boost the interaction towards the ultrastrong regime, as well as to engineer red and blue sideband couplings, nonlinear photon hopping, and classical gauge fields. We discuss how these dynamically tunable superconducting circuits enable key applications in the fields of all-optical quantum computing, continuousvariable quantum information, and quantum simulation - all within the reach of the state of the art in circuit-QED experiments.
\end{abstract}

DOI: 10.1103/PhysRevB.87.134504

PACS number(s): 42.50.Pq, 03.67.Lx, 85.25.-j

\section{INTRODUCTION}

The field of circuit quantum electrodynamics (circuit QED) studies the interaction between artificial atoms and artificial photons, ${ }^{1,2}$ both of them implemented with the same technology: superconducting circuits cooled to millikelvin temperatures. A key feature of these systems is that, based on the same microscopic model, both the photonic degrees of freedom and the artificial atoms have similar energy scales and may interact very strongly. Hence, they show effects which are beyond those explored in the optical domain. A paradigmatic example is the failure of the rotating-wave approximation when the qubitphoton coupling approaches the qubit and photon energies. ${ }^{3,4}$ Aside from the development of qubits and the control of their interactions, ${ }^{5-7}$ circuit QED has recently started to focus on the photons themselves, mostly in the context of two different experimental configurations. In the first type of setup, cavities are replaced with open transmission lines and propagating microwave photons that move and interact with localized qubits. This allows us to study one-dimensional artificial QED, atom-light interaction, ${ }^{8}$ electromagnetically induced transparency, ${ }^{9}$ causality, ${ }^{10}$ and quantum metamaterials ${ }^{11-15}$ and to implement photodetectors ${ }^{16-18}$ and routers. ${ }^{19}$ The other type of setup is based on polariton physics: $:^{20,21}$ by coupling multiple cavity-qubit systems it is possible to build lattices on which dressed photons hop and interact, either attractively or repulsively, implementing Hubbard-type models or spin Hamiltonians. $^{22,23}$ This gives rise to well-known models, such as the Tonks-Girardeau gas; ${ }^{24}$ however, the architecture based on superconducting cavities and Josephson junctions also allows for the exploration of new phenomena, such as gauge fields and frustration. ${ }^{25}$

In this work we reconsider the architecture of coupled superconducting cavities, designing a tunable coupling between nearest-neighbor resonators. The setup that we have in mind consists of an array of linear resonators, directly connected through different types of Josephson junction (JJ) circuits (see Fig. 4). The fixed circuit structure is associated with static, geometry-dependent capacitive and inductive couplings between the resonators, while the interactions created by the JJ circuits can be tuned in amplitude, phase, and form by external magnetic fields.

The coupling elements in this coupled-resonator framework can be operated in two ways: (i) with a stationary configuration of magnetic fields that determines the associated coupling matrix between oscillator modes or (ii) with a periodic multicolor driving that allows for engineering a variety of photon-photon interactions, from red and blue sidebands to gauge fields, passing through correlated photon hopping and Kerr nonlinearities, or simply canceling the usual cross-talk between resonators. In particular, we show how to engineer couplings of the form $\eta_{1} a^{\dagger} b+\eta_{2} a b+$ H.c. between any two resonators $a$ and $b$, with adjustable strengths $\eta_{1,2}$ and possibly complex phases in front of each term.

We must remark that our coupling circuits are based on superconducting quantum interference devices (SQUIDs), those ubiquitous devices which are used for anything from measuring magnetic fields and qubits to parametrically controlling microwave transmission lines. ${ }^{26,27}$ Despite the widespread use of SQUIDS, the framework that we introduce here combines in a single design original solutions to many different problems that were addressed only individually in previous works. First of all, the design we present here is nondispersive. This is in contrast to other proposals based on dispersive couplings via qubits ${ }^{28-30}$ and disconnected SQUID loops. ${ }^{31}$ While in those works the coupling is limited by the dispersive condition, the setup in this work allows us to reach the strong-and ultrastrong-coupling regimes-larger than the corresponding decay rates or comparable to the cavity frequencies, respectively. This demands on our side a careful microscopic derivation that merges ideas from the work of 
Bourassa et al. ${ }^{32}$ with the field of coupled cavities. Second, our SQUID coupling works between separate resonators, allowing for scalable architectures with one- and two-dimensional configurations as shown in Fig. 4. This is in contrast with other works that showed coupling between different modes of the same resonator, ${ }^{33-36}$ or which couple a few nonlinear resonators. ${ }^{37}$ Third, unlike other scalable proposals based on circulator-type devices, ${ }^{25,38}$ our setup is minimalistic and relies on a very robust circuit which is not sensitive to charge noise and does not require a precise balance of junctions. Finally, our design achieves full tunability, regardless of other elements that may coexist with the cavities, such as qubits or magnetic impurities.

As short-term applications of this work we would like to address two fields. The first one has been sketched above: by tuning the coupling between different cavities it is possible to tune the lattice topology, the coupling strength, and even the phase of the hopping terms in polariton arrays. This nicely complements existing proposals which show how to tune the photon nonlinearity by manipulating the qubit inside the cavity ${ }^{22}$ and gives access to effective gauge fields without relying on fragile coupling elements. ${ }^{25}$ The second type of application points along the line of quantum information and the manipulation of continuous-variable states. By means of the coupling circuits in this toolbox one may implement any nearest-neighbor quadratic Hamiltonian with any time dependence and geometry, as far as it is embeddable in a twodimensional (2D) manifold. Notice that our implementations are limited to a frequency scale which is well below the plasma frequency of the SQUID, where it behaves as a passive device. However, this is always true for the purposes of this work. ${ }^{39}$ This Hamiltonian can be used to implement interesting states, such as two-dimensional continuous-variable Gaussian states, ${ }^{40}$ whose tomography could be supplemented by embedded qubits ${ }^{41}$ or moving probes. ${ }^{42}$

The paper is organized as follows. In the first part (Sec. II) we study two superconducting resonators that are close together and subject to a mutual inductive and capacitive interaction. Using the Lagrangian quantization, we will show that, in both the weak- and strong-coupling regimes, the geometric cross-talk gives rise to a constant beam-splitter type of interaction. In the second part of this work (Sec. III) we propose two quantum circuits that dynamically tune the inductive coupling between the resonators. The first one is a SQUID which is galvanically coupled to two resonators. The second one uses instead two coupling wires, creating an interference device between resonators. We will discuss both models analytically, demonstrating that they can tune and switch off the overall resonator-resonator coupling. In Sec. III C we study the validity of our designs under realistic experimental conditions, estimating the coupling strengths that can be attained in current experiments. In Sec. IV A we consider the situation of a time-dependent resonator coupling. We show that a periodic modulation of the coupling makes it possible to engineer sidebands not only in a nonperturbative fashion, ${ }^{43}$ but also in a controlled way, tuning the strength and phase of both the rotating and counterrotating terms. Finally, in Sec. IV C we summarize our results and suggest a large set of potential applications, ranging from quantum information to quantum simulation.

\section{STATIC COUPLING}

In this section we derive the Hamiltonian that rules the dynamics of two coupled superconducting stripline resonators, and give a general expression for the different coupling constants that arise from the model. First we consider the simplest case of coupling, caused by the mutual inductance and mutual capacitance due to the spatial proximity of the resonators. Since the coupling is time independent and determined by the detailed spatial arrangement of the resonators, we refer to it as static geometric coupling. The discussion of this interaction is done for a particular configuration of parallel resonators, but the objective is just to exemplify how this coupling manifests itself as a beam-splitter interaction.

Consider two superconducting stripline resonators of length $2 l$, as depicted in Fig. 1(a). In this particular layout, the coupling occurs mainly within a middle section of length $2 l_{c}$, where the resonators approach each other. Assuming that the cross-talk is given by the mutual inductance $l_{m}$ and mutual capacitance $c_{m}$ induced in this middle region, ${ }^{44,45}$ we can write down the following Lagrangian density:

$$
\mathcal{L}=\sum_{i, j} \int_{-l}^{l}\left[\frac{\hat{c}_{i j}}{2} \dot{\phi}_{i} \dot{\phi}_{j}-\left(\frac{1}{2 \hat{l}}\right)_{i j} \partial_{x} \phi_{i} \partial_{x} \phi_{j}\right] d x,
$$

where both the flux fields $\phi_{i}(x)$ and the capacitance $\hat{c}$ and inductance matrices $\hat{l}$, depend on the position along the transmission line:

$$
\begin{gathered}
\hat{c}=c_{0}(x)+c_{m}(x)\left(\mathbb{I}-\sigma_{x}\right), \\
\hat{l}=l_{0}(x)+l_{m}(x) \sigma_{x},
\end{gathered}
$$

where $\sigma_{x}$ is the Pauli matrix. A full derivation of Eq. (1) can be obtained from the lumped circuit equivalent of the striplines [Fig. 1(b)] and it is thoroughly discussed in Appendix A.

(a)
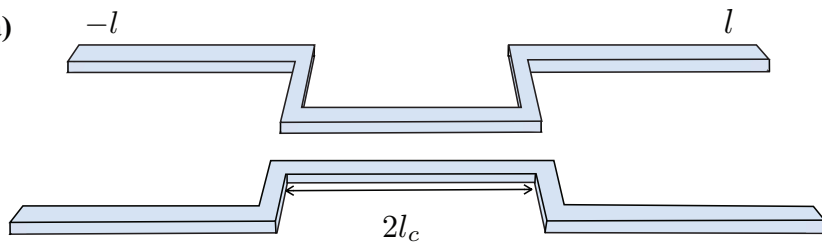

(b)

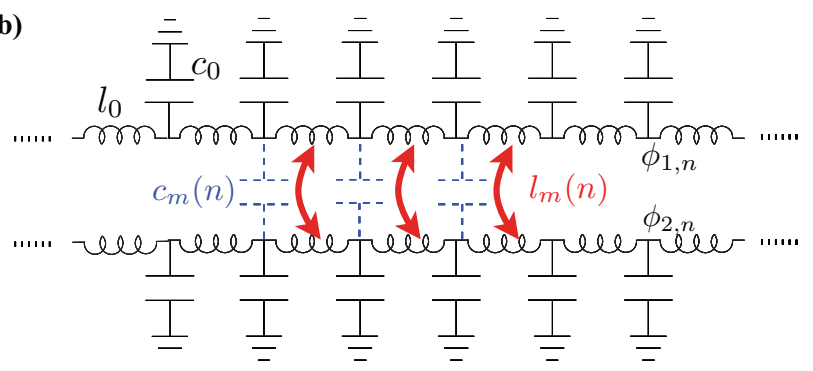

FIG. 1. (Color online) (a) Sketch of the geometrical arrangement of the two coupled superconducting stripline resonators of length $2 l$. A finite interaction is present only in the coupling region of length $2 l_{c}$ and is negligible elsewhere. (b) Schematics for the lumped circuit equivalent. We explicitly draw the mutual capacitances (dashed blue lines) and the mutual inductive coupling (red arrows). The node flux $\phi_{n}$ is also indicated. 
For the sake of simplicity, we will consider the capacitance and inductance per unit length of each line, $c_{0}(x)$ and $l_{0}(x)$, to be constant, and use piecewise-constant functions for the mutual inductance and capacitance:

$c_{m}(x)=\left\{\begin{array}{l}c_{m},|x|<l_{c}, \\ 0 \text { otherwise, }\end{array} \quad l_{m}(x)=\left\{\begin{array}{l}l_{m},|x|<l_{c}, \\ 0 \text { otherwise. }\end{array}\right.\right.$

We derive a normal-mode expansion for the flux $\phi_{j}(x, t)=$ $\sum_{n} q_{j, n}(t) u_{n}(x)$ in each resonator $j=1,2$. In what follows we restrict ourselves to the fundamental mode of each resonator with frequency $\omega_{0}$ and total capacitance $C_{r}=\int_{-l}^{l} c_{0}(x) d x$. This is always true if we remain in the strong-coupling limit, where $g \ll \omega_{1}-\omega_{0}$, or we work with zero-mode resonators, like the ones used in Ref. 4. Within this subspace and mode expansion, the interaction term gives rise to off-diagonal terms, as expected from an interaction between two cavities, but also diagonal terms that induce a renormalization (dressing) of the oscillator frequencies. This dressed resonance frequency is

$$
\omega=\omega_{0} \sqrt{\frac{1+C}{1+2 C}\left(1+\frac{1}{v} \frac{L^{2}}{1-L^{2}}\right)},
$$

expressed in terms of two overlap integrals

$$
\Delta_{1}=\int_{-l_{c}}^{l_{c}}\left[u_{0}(x)\right]^{2} d x, \quad \Delta_{2}=\int_{-l_{c}}^{l_{c}}\left[\partial_{x} u_{0}(x)\right]^{2} d x,
$$

where $C=c_{m} \Delta_{1} / C_{r}, L=l_{m} / l_{0}$, and $v=\omega_{0}^{2} C_{r} l_{0} / \Delta_{2}$ is a geometric factor.

We finally proceed with the quantization of this model, introducing the oscillator length $a_{0}=$ $\sqrt{\hbar(1+C) / C_{r} \omega(1+2 C)}$. We express the phase-space operators in terms of the Fock operators $q_{j}=a_{0}\left(a_{j}+a_{j}^{\dagger}\right) / \sqrt{2}$ and $p_{j}=i \hbar\left(a_{j}^{\dagger}-a_{j}\right) / \sqrt{2} a_{0}$. This leads to

$$
\begin{aligned}
H= & \hbar \omega \sum_{j=1,2} a_{j}^{\dagger} a_{j}-\hbar g_{c}\left(a_{1}^{\dagger}-a_{1}\right)\left(a_{2}^{\dagger}-a_{2}\right) \\
& -\hbar g_{i}\left(a_{1}^{\dagger}+a_{1}\right)\left(a_{2}^{\dagger}+a_{2}\right) .
\end{aligned}
$$

The coupling constants $g_{c}$ and $g_{i}$ account for the static capacitive and inductive contributions to the coupling, respectively,

$$
\begin{gathered}
g_{c}=\frac{\omega_{0}}{2} \sqrt{\frac{C^{2}}{(1+C)(1+2 C)}\left(1+\frac{1}{v} \frac{C^{2}}{1-C^{2}}\right)}, \\
g_{i}=\frac{\omega_{0}}{2} \frac{1}{v} \frac{L}{1-L^{2}} \sqrt{\frac{1+C}{1+2 C} \frac{1}{1+\frac{1}{v} \frac{L^{2}}{1-L^{2}}}} .
\end{gathered}
$$

The usual limits in quantum optics correspond to the weak-coupling and strong-coupling regimes. In both of them $g_{c, i} / \omega_{0} \ll 1$, so that the frequency renormalization becomes negligible (provided that $C, L \ll 1$ ). We can then invoke the rotating-wave approximation (RWA) and transform (7) to the beam-splitter model,

$$
H \simeq \hbar \omega_{0} \sum_{j=1,2} a_{j}^{\dagger} a_{j}-\hbar\left(g_{i}-g_{c}\right)\left(a_{1}^{\dagger} a_{2}+a_{2}^{\dagger} a_{1}\right) .
$$

Note how the resulting Hamiltonian can be interpreted as an exchange or hopping of excitations between modes, similar to optical lattice and tight-binding models.
This type of static geometric coupling is implicit in the experimental configurations of coupled-cavity models, ${ }^{20,21}$ although previous designs have inclined to consider a capacitive coupling taking place at the electric field nodes (current antinodes) of the resonator, ${ }^{22,25,38}$ sometimes enhanced by an additional $\mathrm{JJ}$ circuit. ${ }^{25,38}$

\section{TUNABLE COUPLING}

In this section we study alternative mechanisms for coupling two or more linear resonators. On the one hand we aim at a larger coupling strength, and on the other hand we wish to achieve real-time tunability of the couplings. For both goals it will be advantageous to rely on inductive rather than capacitive coupling. First of all, the inductive coupling realized by JJs and loops intersected by JJs (e.g., SQUIDs) can be tuned by an applied magnetic field varying the magnetic flux threading the JJs or loops. Second, and equally important, inductive interactions can be enhanced, profiting both from the kinetic inductance of thin superconducting films and from embedded junctions working in the linear regime. ${ }^{32}$ Based on the two previous ideas, we envision the two coupling elements sketched in Fig. 2. We will study these designs analytically, deriving expressions for the effective interactions and coupling strengths.

\section{A. The SQUID as a coupler}

Given the large inductance provided by the Josephson junctions, one could naively think of connecting both cavities with a superconducting wire interrupted by a Josephson junction. In doing so one would achieve a static ultrastrong coupling. However, for tuning the Josephson inductance of a single junction we have to generate a magnetic flux of the order of a flux quantum threading the junction area. Due to the small junction area unpractically large magnetic fields would be required. Fortunately, we can design a much better tunability by using a SQUID configuration, as depicted in

(a)

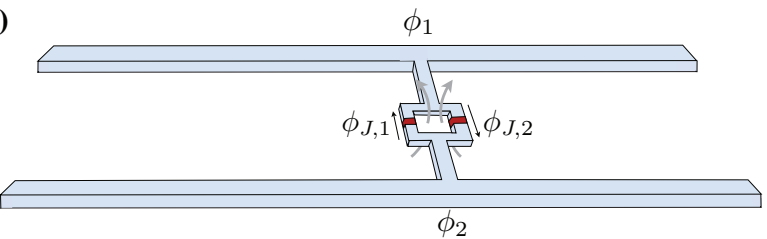

(b)

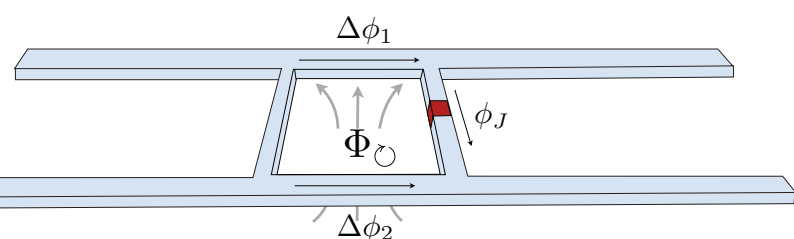

FIG. 2. (Color online) Architectures leading to a tunable microwave beam splitter. (a) A dc SQUID (superconducting loop intersected by two Josephson junctions) mediates the coupling between two stripline resonators (ground planes not shown). The established pointlike contact between the resonators takes the coupling to the ultrastrong domain. (b) A superconducting ring intersected by a Josephson junction now partially shares its branches with the cavities, improving the switching capability. 
Fig. 2(a). Since the SQUID loop area is much larger than the junction area, much smaller control fields are required. Note that the use of the dc SQUID to achieve fast tunability is quite common in circuit QED. For instance, they have been used to very rapidly change the effective length of resonators ${ }^{46}$ to observe the dynamical Casimir effect, ${ }^{26,27}$ and through similar principles they underlie the basis of recent Josephson parametric amplifiers. ${ }^{47-49}$ More related to our work, SQUIDs have also been suggested as a coupling element for flux qubits, ${ }^{50}$ using an external flux to control their mutual inductance. However, the peculiarities of our setup, which deals with continuous variables and has subtle differences, merits a separate discussion below.

A short line with the SQUID as depicted in Fig. 2(a) represents a small contribution to the original Lagrangian density (1). Following Ref. 51 we compute $\mathcal{L}_{t}=\mathcal{L}+\mathcal{L}_{\text {SQUID }}$ with

$$
\mathcal{L}_{\text {SQUID }}=\sum_{k=1}^{2} \frac{C_{J, k}}{2} \dot{\phi}_{J, k}^{2}+E_{J, k} \cos \left(\frac{2 \pi \phi_{J, k}}{\Phi_{0}}\right),
$$

where $\phi_{J, k}$ represent the flux differences along the junctions $k=1,2$. We use fluxoid quantization along the SQUID loop, $\phi_{J, 1}+\phi_{J, 2}+\Phi_{\circlearrowright}=n \Phi_{0}$, to express the Lagrangian in terms of the variables $\phi_{ \pm}=\frac{1}{2}\left(\phi_{J, 1} \pm \phi_{J, 2}\right)$ and the total flux enclosed by the loop, $\Phi_{\circlearrowright}$. For simplicity, we assume $\Phi_{\circlearrowright} \simeq \Phi_{\text {ext }}$, that is, we are neglecting the additional flux generated by the circulating loop current. This is equivalent to restricting our discussion to screening parameters $\beta_{L}=2 \pi L I_{c} / \Phi_{0}<1$ as discussed in more detail below. Here, $L$ is the loop inductance and $I_{c}$ the critical current of the Josephson junctions. If the SQUID is symmetric, $C_{J, 1}=C_{J, 2}$ and $E_{J, 1}=E_{J, 2}$, the coupling becomes $E_{\text {eff }} \cos \left(\pi \phi_{-} / \Phi_{0}\right)$, with an effective Josephson coupling energy that depends on the flux threading the SQUID loop,

$$
E_{\text {eff }}=2 E_{J} \cos \left(\pi \Phi_{\circlearrowright} / \Phi_{0} a\right) .
$$

The voltage-phase relation $\dot{\phi}_{-}=\dot{\phi}_{1}(x)-\dot{\phi}_{2}(x)$ allows us to express $\phi_{-}$in terms of the voltages at the edges of the connecting wire. In the linear limit of small fluxes, i.e., small photon number (see Appendix B), we can write a quadratic coupling between fields,

$$
\mathcal{L}_{\mathrm{SQUID}} \simeq C_{J}\left(\dot{\phi}_{1}-\dot{\phi}_{2}\right)^{2}-\frac{2 \pi^{2} E_{\mathrm{eff}}}{\Phi_{0}^{2}}\left(\phi_{1}-\phi_{2}\right)^{2},
$$

which by means of the normal-mode decomposition adopts the form of Eq. (7). The cross-term of Eq. (13) adds up to the static inductive coupling (9), making it tunable. This tunability relies on the fact that the Josephson energy $E_{\text {eff }}$ is flux dependent, ${ }^{52}$ and thus

$$
g_{i}=g_{i}^{\text {static }}+\frac{4 \pi^{2} \hbar}{\Phi_{0}^{2} C_{r} \omega} E_{J} \cos \left(\pi \Phi_{\circlearrowright} / \Phi_{0}\right)
$$

in Eq. (10) can be changed in magnitude and sign. For an appropriate value of the external flux (close to $\Phi_{\circlearrowright}=\Phi_{0} / 2$ if $\left.\left|g_{c}\right| \ll\left|g_{i}\right|\right)$ we can fully deactivate the coupling. Note that in this treatment the SQUID variables are not independent and introduce no new modes: the fluxoid quantization allows us to express them in terms of the cavity modes. This topic and the linearization are discussed in more detail in Appendix B 3 using exact diagonalizations.

We finally note that our setup is robust against small differences in the two junction energies, $E_{J 1,2}=E_{J}(1 \pm \varepsilon)$. In this case one can still expand $\phi_{1,2}=\frac{1}{2} \Phi_{\circlearrowright} \pm \phi_{-}$, linearizing around $\phi_{-} \simeq 0$ to obtain

$$
\mathcal{L}_{\mathrm{SQUID}} \pm 2 \varepsilon E_{J} \sin \left(\pi \Phi_{\circlearrowright} / \Phi_{0}\right) \frac{2 \pi \phi_{-}}{\Phi_{0}}
$$

The linear term in this equation amounts just to a displacement of the oscillators and does not add up to the total coupling, preserving the tunability of the setup. We will use this idea in the following setup.

\section{B. Superconducting ring coupler}

The second design is shown in Fig. 2(b). It consists of a superconducting ring interrupted by a single Josephson junction. Since both resonators share a branch of the loop that couples them, the Lagrangian acquires new contributions with the kinetic inductance of the superconductor. ${ }^{32}$ This kinetic coupling can be very strong, while still retaining the switching capability due to the fluxoid quantization inside the loop, similar to previous designs for superconducting qubits. ${ }^{53}$

Note that in comparison to previous studies of SQUIDmediated entanglement between cavities, ${ }^{54-56}$ this setup is much simpler because there are no degrees of freedom for the SQUID: the flux through the SQUID is the same as the flux to the cavities. Additionally, this design profits from the stronger interaction due to the galvanic coupling of the circuit elements.

Our derivation is based on two nonessential constraints. The first one is that the loop is small enough for its selfinductance to be neglected $\left(\beta_{L} \ll 1\right)$. The second one is that the wire without junction touches the resonators at points where the flux of the coupled modes is zero, ${ }^{57} u_{0}\left(x_{1}\right)=0$. Under these circumstances the coupling term reads

$$
\mathcal{L}_{J J}=\frac{C_{J}}{2} \dot{\phi}_{J}^{2}+E_{J} \cos \left(\frac{2 \pi \phi_{J}}{\Phi_{0}}\right) .
$$

The fluxoid quantization inside the loop, $\Delta \phi_{2}-\Delta \phi_{1}+\phi_{J}=$ $-\Phi_{\circlearrowright}$, allows us to get rid of the flux variable $\phi_{J}$ and rewrite the coupling in terms of the branch fluxes $\Delta \phi_{1,2}$,

$$
\begin{aligned}
\mathcal{L}_{J J}= & \frac{C_{J}}{2}\left(\Delta \dot{\phi}_{1}-\Delta \dot{\phi}_{2}\right)^{2} \\
& +E_{J} \cos \left[\frac{2 \pi\left(\Delta \phi_{1}-\Delta \phi_{2}+\Phi_{\circlearrowright}\right)}{\Phi_{0}}\right] .
\end{aligned}
$$

At this point we will repeat the linearization of the cosine, much as in Eq. (13). However, now the Taylor expansion will depend on the external flux $\Phi_{\circlearrowright}$, producing linear and quadratic contributions of different magnitude. We start with the normalmode decomposition of the branch fluxes and restrict ourselves to the lowest-energy modes

$$
\Delta \phi_{j}=\phi_{j}\left(x_{2}\right)-\left.\phi_{j}\left(x_{1}\right) \simeq q_{j, 0} \partial_{x} u_{0}(x)\right|_{x_{1}} \Delta x .
$$


Substituting these terms in Eq. (17) produces the quadratic Lagrangian for the fundamental modes,

$$
\begin{aligned}
L_{J J}= & \frac{1}{2} \sum_{j=1,2}\left(\alpha_{J} \dot{q}_{j}^{2}-\beta_{J} q_{j}^{2}\right) \\
& +\gamma_{J}\left(q_{1}-q_{2}\right)-\alpha_{J} \dot{q}_{1} \dot{q}_{2}+\beta_{J} q_{1} q_{2} .
\end{aligned}
$$

The expressions for all coefficients can be computed from first principles,

$$
\begin{gathered}
\alpha_{J}=C_{J}\left[\partial_{x} u_{0}(x=0)\right]^{2} \Delta x^{2}, \\
\beta_{J}=E_{J} \frac{4 \pi^{2}}{\Phi_{0}^{2}}\left[\partial_{x} u_{0}(x=0)\right]^{2} \Delta x^{2} \cos \left(\frac{2 \pi \Phi_{\circlearrowright}}{\Phi_{0}}\right), \\
\gamma_{J}=E_{J} \frac{2 \pi}{\Phi_{0}}\left[\partial_{x} u_{0}(x=0)\right] \Delta x \sin \left(\frac{2 \pi \Phi_{\circlearrowright}}{\Phi_{0}}\right),
\end{gathered}
$$

where we have assumed that the superconducting loop is placed around $x=0$. Of these terms, $\gamma_{J}$ is a linear displacement of the cavity eigenmodes and does not transfer energy. The capacitive and inductive terms $\alpha_{J}$ and $\beta_{J}$ are the only ones that contribute to the intercavity coupling $g_{c}$ and $g_{i}$, and to the frequency renormalization. More precisely, we obtain the model (7) with mode frequency

$$
\omega=\omega_{0} \sqrt{1+\frac{\beta_{J}}{C_{r} \omega_{0}^{2}}}
$$

and coupling strengths

$$
g_{i}=g_{i}^{\text {static }}+\frac{\beta_{J}}{2 C_{r} \omega}, \quad g_{c}=g_{c}^{\text {static }}+\frac{\alpha_{J} C_{r} \omega}{2} .
$$

In general we will find that for a junction that works in the flux regime the term $\beta_{J}$ dominates all other contributions. But even without this assumption, it is true that while $g_{c}$ is fixed, the value of $g_{i}$ depends entirely on $\beta_{J}$ and can be changed in magnitude and sign, either enhancing the strength of the beam-splitter coupling (10), or switching it off entirely for a value of $\Phi_{\circlearrowright} \simeq \Phi_{0} / 4$.

While the coupling strength grows with the loop size $\Delta x$, we cannot make it arbitrarily large because then we are no longer allowed to neglect the additional flux $\phi_{L}$ caused by the circulating loop current due to the increasing value $L$ of the self-inductance of the loop. ${ }^{58}$ In this case the total flux threading the loop is given by the sum of the external flux $\Phi_{\text {ext }}$ and the flux $\Phi_{L}$. However, as explained in Ref. 58, Chap. 8.4, provided that

$$
\beta_{L}=\frac{2 \pi L I_{c}}{\Phi_{0}}<1
$$

we can ensure that the $\Phi_{\circlearrowright}$ versus $\Phi_{\text {ext }}$ dependence is single valued, allowing us full tunability of the coupling. This condition means that the maximum loop current $I_{c}$ cannot generate more than a single flux quantum. It restricts us to loop sizes of around 5\% of the resonator length. We now study various methods to increase the coupling strength while preserving the condition above.

\section{Estimation of the coupling strength}

We are interested in an upper bound for the coupling strength $g$. More precisely we would like to access both the strong- and ultrastrong-coupling regimes. Strong coupling means that it is possible to observe Rabi oscillations between the two cavities because the coupling $g$ is larger than the resonator decay rate $\kappa$. On the other hand, ultrastrong coupling occurs when the RWA fails, which in this case implies that the number of photons in the ground state, which is proportional to $g / \omega$, approaches 1 .

Looking at the first proposal [see Fig. 2(a) and Sec. III A] we note that the maximum coupling is reached for an external flux $\Phi_{\circlearrowright}=n \Phi_{0}$ threading the SQUID loop, and thus yielding

$$
g_{i} \simeq \frac{4 e^{2}}{2 C_{r}} \frac{E_{J}}{\hbar^{2} \omega}\left|u_{0}(x)\right|^{2}=\frac{\pi I_{c}}{\Phi_{0}} Z\left|u_{0}(x)\right|^{2},
$$

where $I_{c}$ is the critical current of the junction, $Z$ is the resonator impedance, and the eigenmode $u_{0}(x)$ satisfies $0<\left|u_{0}(x)\right|<$ $\sqrt{2}$. To preserve the power field expansion, we suppose the SQUID to be built at a position such that $\left|u_{0}(x)\right| \leqslant 0.1$ (see Sec. IV B). Under this condition, and using a critical current $I_{c} \simeq 5 \times 10^{-6} \mathrm{~A}$, together with $Z=50 \Omega$, it would be possible to reach a coupling strength up to $g_{i} \simeq 1.2 \mathrm{GHz}$.

On the other hand, for the second proposal [see Fig. 2(b) and Sec. III B] the coupling (24) in the loop becomes

$$
g_{i} \simeq E_{J}\left(\frac{2 \pi}{\Phi_{0}} \frac{\partial \psi(x)}{\partial x} \Delta x\right)^{2},
$$

with $\partial_{x} \psi=\partial_{x} u \sqrt{\frac{\hbar}{2 C_{r} \omega}}$. For a homogeneous resonator [see (A 1)], we can straightforwardly assess the slope of $u_{0}(x)$, finding an exact expression for $g$ :

$$
g=\pi^{2} \frac{\omega_{J} \omega_{c}}{\omega_{0}}\left(\frac{\Delta x}{2 l}\right)^{2},
$$

where $\omega_{J}=E_{J} / \hbar, \omega_{c}=E_{C} / \hbar=(2 e)^{2} / 2 \hbar C_{r}$ is the characteristic charging frequency of the resonator, and $\omega_{0}$ is the firstmode frequency. Using available values for $\mathrm{Nb}$ striplines and junction parameters, we find that the homogeneous resonator remains in the weak-coupling regime, as we envisioned before. For a loop size $\Delta x$ of $1 \%$ of the resonator length, we obtain $g \simeq 2 \mathrm{MHz}$ which represents $0.03 \%$ of the resonator frequency $\omega$ (see Fig. 3).

Adding a constriction to the central part of the resonator increases the field slope and thus the coupling. To this end, for a suitable $\mathrm{Nb}$ inhomogeneous transmission line resonator, ${ }^{32}$ this enhances the coupling up to $g \simeq 100 \mathrm{MHz}$, or equivalently to $1.8 \%$ of the resonator frequency (Fig. 3, red dashed line).

Finally, the coupling can be further enhanced by interrupting the transmission line with a Josephson junction. Due to the presence of the junction, the flux eigenmode presents a constant phase slip $\Delta \phi_{0}$ at $x=0$, which depends on the Josepshon coupling energy of the junction. ${ }^{32,59}$ This additional phase jump enhances the coupling as follows:

$$
g_{i} \simeq E_{J}\left(\frac{2 \pi}{\Phi_{0}} \frac{\partial \psi(x)}{\partial x} \Delta x+\Delta \phi_{0}\right)^{2} .
$$

Optimal parameters for the junction attached to the resonator ( $E_{J \text { res }} \simeq 7 E_{J \text { loop }}$ ) could lead to extremely large couplings of around $g \sim 600 \mathrm{MHz}(9 \%$ of $\omega)$.

The previous numbers have to be compared with similar figures from other setups, such as a circulator-based coupling 


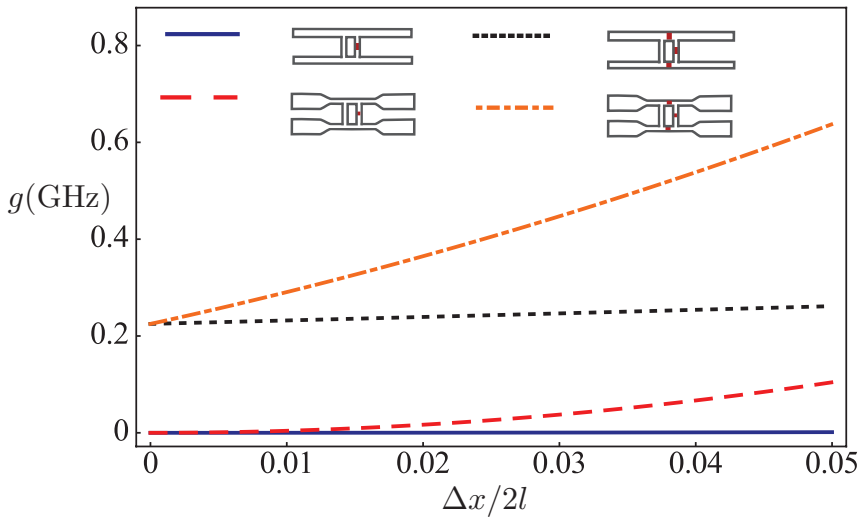

FIG. 3. (Color online) Coupling strength for differerent niobium transmission lines, as a function of the loop size. While homogeneous resonators (blue) hardly reach the strong-coupling regime, inhomogeneous ones (red dashed) do. The coupling strength can be further increased with a Josephson junction interrupting the center conductor, as shown for the homogeneous case (dotted) and the inhomogeneous one (dot dashed). We have considered for each resonator a frequency $\omega_{0} / 2 \pi=6.65 \mathrm{GHz}$.

between resonators, ${ }^{25,38}$ or the dispersive coupling based on SQUID loops ${ }^{31}$ or qubits. ${ }^{28}$ In these three cases the coupling element works with a high detuning $\Delta$ from the resonator frequency. This means that the effective coupling takes the form $g^{2} / \Delta$, where $g$ is the coupling of one resonator to the circulator, SQUID loop, or qubit, $\Delta$ is the detuning from the resonator frequency and the dispersive condition $g \ll \Delta<\omega$ limits the maximum achievable strength to a few percentage points of $\omega$.

\section{APPLICATIONS}

\section{A. Sidebands}

So far we have discussed the possibility of tuning the coupling strength between two resonators, constructing a classical switch that allows us to control the exchange of photons. In this section we discuss a second type of tunability, which consists of engineering an arbitrary linear type of coupling between two resonators:

$$
H_{\mathrm{int}}=g_{1} e^{i \phi_{1}} a^{\dagger} b+g_{2} e^{i \phi_{2}} a b+\text { H.c. },
$$

represented by the Fock operators $a$ and $b$. This would enlarge the applicability of our setup, extending it to the realization of almost any quadratic model with nearest-neighbor interactions.

In order to demonstrate that this is possible we start our discussion by noting that both the dc SQUID and the ring coupler provide us with a flux-dependent coupling

$$
H=\hbar \omega_{a} a^{\dagger} a+\hbar \omega_{b} b^{\dagger} b+g\left(\Phi_{\circlearrowright}\right)\left(a^{\dagger}+a\right)\left(b^{\dagger}+b\right) .
$$

If we now engineer the two resonators to have very different frequencies $\omega_{a}$ and $\omega_{b}$, the static coupling $|g| \ll \omega_{a, b}$ will be effectively suppressed, giving rise to a small dispersive term

$$
H \sim \hbar \omega_{a} a^{\dagger} a+\hbar \omega_{b} b^{\dagger} b+\frac{g^{2}}{\left|\omega_{b}-\omega_{a}\right|} a^{\dagger} a b^{\dagger} b .
$$

However, if we allow for a two-tone driving of the coupling,

$$
\begin{aligned}
g(t)=g\left[\Phi_{\circlearrowright}(t)\right]= & g_{1} \cos \left[\left(\omega_{b}-\omega_{a}\right) t+\phi_{1}\right] \\
& +g_{2} \cos \left[\left(\omega_{a}+\omega_{b}\right) t+\phi_{2}\right],
\end{aligned}
$$

then this driving effectively activates the rotating and counterrotating terms, with the phases given above. To show this we switch to an interaction picture with respect to the two harmonic oscillators,

$$
H_{I}=g(t)\left(a^{\dagger} b e^{i\left(\omega_{a}-\omega_{b}\right) t}+a b e^{-i\left(\omega_{a}+\omega_{b}\right) t}+\text { H.c. }\right) .
$$

The oscillating terms in Eq. (33) will precisely cancel the ones in the previous time-dependent Hamiltonian, leaving behind some other nonresonant terms which act only in higher-order perturbation theory, $O\left(g^{2} / \omega\right)$. The result should be the desired combination of sidebands

$$
H_{\mathrm{eff}}=g_{1} a^{\dagger} b e^{i \phi_{1}}+g_{2} a b e^{i \phi_{2}}+\text { H.c. }
$$

It is worth mentioning that the previous sideband engineering is not perturbative: while we still need to impose the requirement that $\left|g_{1,2}\right| \ll\left|\omega_{b}-\omega_{a}\right|$, the resulting coupling is larger than the dispersive term. This strong coupling and the individual tuning of photon terms would be a wonderful tool to explore the different phase transitions predicted for exotic Bose-Hubbard models, as discussed for instance in Ref. 60.

Another very important feature of the two-tone driving method is that it allows us to control the phases of the rotating and counterrotating terms, for this is related to the phase of the two-tone driving. As we discuss below, this is a very important property, as it allows us to implement effective gauge fields that control the hopping of photons between resonators. Moreover, we achieve this effect by a simple driving of a standard SQUID, without the need of time-reversal symmetry-breaking circuits which might be very sensitive to other noise sources. ${ }^{25}$

Finally, even though the realization of the time dependence (33) might seem complicated, in practice we do not need to tune the flux in a very complicated manner. A simple driving of $\Phi_{\circlearrowright}(t) \simeq \Phi+\delta \Phi \cos (\omega t)$, when introduced in the sinusoidal coupling (14) $g \simeq \cos \left(2 \pi \Phi_{\circlearrowright} / \Phi_{0}\right)$ produces, via the Jacobi-Anger expansion,

$$
\begin{aligned}
g(t) \simeq & \cos \left(2 \pi \Phi / \Phi_{0}\right) J_{0}(\delta \Phi) \\
& +\sin \left(2 \pi \Phi / \Phi_{0}\right) J_{1}(\delta \Phi) \cos (\omega t)+\cdots
\end{aligned}
$$

in terms of the Bessel functions $J_{0}$ and $J_{1}$. This series contains the basic driving plus higher harmonics which will be spectrally suppressed in the coupling term. Alternatively, a suitable dependence for $\Phi_{\circlearrowright}$ can be engineered with around $0.1 \mathrm{~ns}$ resolution using appropriate signal generators. ${ }^{61}$ Again, out of this signal only the resonant terms, with frequencies around $\omega_{a} \pm \omega_{b}$ will contribute to the coupling. Discretization errors in the signal, and higher harmonics, will be averaged out.

\section{B. Nonlinear photon hopping}

So far we have worked with the Josephson junctions in the linear regime, neglecting higher-order terms, which are of the order $\frac{1}{24} E_{J}\left(2 \pi \phi / \Phi_{0}\right)^{4}$. This approximation is valid only when the argument of the trigonometric functions, $2 \pi \phi / \Phi_{0}$, is small, a condition which can be recast as a restriction on the 
number of photons that can populate the resonator. Roughly, for the SQUID we have the condition

$$
\phi \sim u(x) \sqrt{\frac{\hbar Z}{2} n} \ll \frac{\Phi_{0}}{2 \pi}=\frac{\hbar}{2 e},
$$

where $Z$ is the impedance of the resonators, $n$ is the average number of photons, and $u(x)$ is the mode wave function at the coupling points. Using, in the same way as above, the value $u(x)=0.1$ restricts the number of photons to $n<1000$ (see Appendix B 1), which does not represent a restriction for the few photon applications that we envision.

The question now is what happens when we do not neglect the nonlinear terms. In this case we have the potential to introduce new interactions between resonators, which are now of higher order and include on-site nonlinearities $n_{i}^{2}$, nearestneighbor attractive or repulsive interactions $n_{i} n_{j}$, photon-pair hopping $a_{i}^{\dagger 2} a_{j}^{2}$, etc. (see Appendix B 2). Of these terms some are already strongly suppressed because of being off resonant; this is the case for interactions with odd powers, such as $a_{i}^{\dagger 3} a_{j}$. The Kerr nonlinearities will always be present and give rise to extended Bose-Hubbard physics. Finally, the correlated hopping terms $a_{i}^{\dagger 2} a_{j}^{2}$ can be resonantly enhanced using the same technique that we employ for the sidebands: introducing a frequency mismatch between neighboring cavities and driving with exactly the frequency which is needed to select this process, $2\left(\omega_{i}-\omega_{j}\right)$. With all these tools we envision the possibility of engineering very interesting models, such as a condensate of pairs of photons, ${ }^{62}$ which are very hard to engineer in other systems.

\section{Outlook and discussion}

Summing up, in this work we have studied two different ways to engineer the coupling between superconducting resonators: one is geometric and static in nature, while the other relies on nonlinear coupling circuits and can be easily tuned in and out of the strong-coupling regime. Both elements together form a powerful toolbox for implementing almost arbitrary models consisting of a low-dimensional (from 1D to 2D) array of resonators with tunable nearest-neighbor interactions, as in the model sketched in Fig. 4. Let us now discuss some of the potential applications of such circuits.

\section{Traditional quantum optics}

The implementation of tunable sidebands in coupled resonators opens the door to many well-known processes from quantum optics. Some of them are the squeezing of different modes via those sidebands, frequency conversion of photons as they are transferred between cavities, parametric generation of photons via $a^{\dagger} b^{\dagger}+a b$ terms, entanglement production at high temperatures, ${ }^{63}$ etc. The beam-splitter Hamiltonian is also the cornerstone of all-optical quantum information processing, as suggested in Ref. 37 for a different circuit-QED architecture.

\section{Harmonic models}

The most immediate application of our design would be to implement arbitrary quasilocal and quadratic Hamiltonians, with the aim of studying the dynamical or static properties of many-body Gaussian states. This includes a variety of
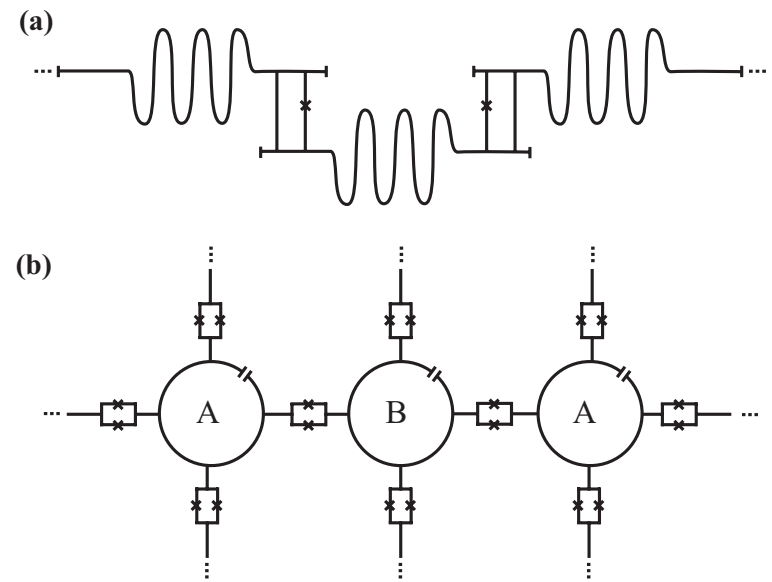

FIG. 4. (a) One-dimensional array of cavities coupled by means of a superconducting ring coupler. (b) Two-dimensional lattice of circular resonators, coupled by dc SQUIDs. Both lattices are bipartite. Using different resonator frequencies for each sublattice, $\omega_{A} \neq \omega_{B}$, we can use the techniques from Sec. IV A to engineer any sideband interaction between the arrays.

studies, such as the static correlations in the model ${ }^{64}$ and their relation to the underlying entanglement, dynamical quantum phase transitions from trivial to critical phases, the study of propagation of correlations in nonequilibrium models and their relation to Lieb-Robinson bounds, ${ }^{65-67}$ etc. In this context, the tunability of the coupling plays two different roles. On the one hand it allows us to change the parameters of the Hamiltonian in an abrupt or smooth way, for instance to study a dynamical quantum phase transition or a quench. On the other hand and equally important, by switching off all couplings we can freeze the quantum state of the oscillators, giving us time to measure the properties of the system, either with different measurement qubits or through a movable probe. ${ }^{42}$

\section{Anharmonicity and thermalization}

The interest of the harmonic problems lies in their simplicity and the possibility of obtaining analytical and numerical results for different geometries and sizes. However, as soon as we introduce a small nonlinearity in our system, we can say very little about their dynamical and static properties and many of the simulations which we mentioned in the previous paragraph become open problems. In particular, one very simple problem which deserves being studied is that of thermalization. The basic idea is to replace the linear resonators in Fig. 4 with resonators that host a tunable and weak nonlinearity in the form of a SQUID (similar to Ref. 27 but outside the linear regime). One would then prepare the ground state of the cavities with a value of the coupling, and then abruptly quench this coupling to a different (larger or smaller) value in which the prepared state is not a ground state. Throughout this process it will be possible to track the relaxation of the oscillator chain or lattice, studying how its behavior is modified by the presence of the nonlinearity. Note that it is well known that the simulation of such time-dependent many-body problems becomes numerically intractable for a few resonators. For instance, assuming a truncation basis with ten photons per cavity, a state of ten 
coupled cavities requires already 10 gigabytes of memory. But the problem of time evolution is worse, for truncation and rounding errors introduce a very fast growth of errors such that after a short runaway time, even the most sophisticated density matrix renormalization group (DMRG) many-body simulation methods ${ }^{68}$ provide only qualitative results. ${ }^{69}$ In this sense, having good quantum simulators would be an invaluable tool to advance the understanding of these nonequilibrium processes beyond those regimes which can be simulated with more straightforward methods. ${ }^{72}$

\section{Coupled cavities and gauge fields}

Along the lines of anharmonic systems, another interesting problem is the study of coupled cavities or Jaynes-Cummings lattices. $^{20,21}$ The setup would be that of Fig. 4, but with one qubit attached to each resonator. The coupled qubit-resonator system behaves as a highly nonlinear element, implementing a quasiparticle known as a "polariton," which may hop from resonator to resonator through our tunable coupling elements. This can be roughly formulated as a Bose-Hubbard Hamiltonian

$$
H=\sum_{i j} t_{i j} a_{i}^{\dagger} a_{j}+U\left(a_{i}^{\dagger} a_{i}\right)
$$

with a very nonlinear on-site interaction $U$ and a hopping $t_{i j}$ which, unlike in previous proposals, ${ }^{22}$ is now dynamically tunable. This allows us to explore the quantum phase transitions from weak interactions $U \ll|t|$ to hard-core particles $U \gg|t|$ simply by reducing the hopping instead of arbitrarily boosting the qubit-resonator interaction-something which might be more challenging from the theoretical and experimental point of view.

In addition to the usual Mott-superfluid phase transition, we now have control over the phase of the hopping, $t_{i j}=$ $|t| \exp i \theta_{i j}$. The procedure, as described in the previous section, consists of engineering two coupled sublattices $[A$ and $B$ in Fig. 4(b)] of resonators with different frequencies, $\omega_{A} \neq \omega_{B}$. Applying a multitone driving on the bonds that connect the two sublattices, we can create an array of phases $\theta_{i j}$ which have a nontrivial flux around each plaquette. This will allow us to probe integer quantum Hall physics with polaritons, ${ }^{25}$ without the use of circulators.

In summary, we have shown that in circuit QED, tunable coupling between resonators can be implemented via simple Josephson circuits. We have developed this initial idea into a profound theoretical basis for exciting multiresonator experiments ranging from arbitrary sideband interactions to setups scalable towards the many-body regime. On the theoretical side, our results lend themselves to being expanded to advanced scenarios, such as the relation between our circuit models and Josephson junction arrays, the influence of decoherence, or even the design of models with tunably dissipative elements.

\section{ACKNOWLEDGMENTS}

We thank Juan José Mazo for useful discussions. This work was supported by the EU Projects PROMISCE and CCQED, Spanish MICINN Projects No. FIS2009-10061 and No. FIS2011-25167, and CAM Research Consortium
QUITEMAD No. S2009-ESP-1594. B.P. acknowledges financial support from CSIC Contract JAE-PREDOC2009. F. W., F. D., A. M., and R. G. acknowlegde support by the German Research Foundation via SFB 631 and the German Excellence Initiative via the Nanosystems Initiative Munich (NIM).

\section{APPENDIX A: LUMPED ELEMENT MODEL OF TWO COUPLED RESONATORS}

Here we derive the density Lagrangian (1) of Sec. II from the quantum network theory perspective. The appendix is divided into three parts: in the first one we review the quantization of a single microstrip resonator. In the second one, we consider the equivalent circuit of the coupled striplines shown in Fig. 1(a) in its lumped element model [see Fig. 1(b)]. The Kirchhoff equations derived here will give rise to the Lagrangian (1) in the continuum limit.

\section{Single-oscillator description}

Here, we detail the description for the single-resonator case. The transmission line field equations are obtained from their lumped circuit equivalent. Neglecting losses, it can be described as a series of $L C$ circuits. ${ }^{58}$ In the continuum limit, the resulting field equations can be obtained from the Lagrangian

$$
\mathcal{L}_{0}=\frac{1}{2} \int_{-l}^{l} d x\left[c_{0}(x) \dot{\phi}(x, t)^{2}-l_{0}(x)^{-1} \partial_{x} \phi(x, t)^{2}\right],
$$

where $c_{0}(x)$ and $l_{0}(x)$ are the capacitance and inductance per unit of length, respectively; otherwise, $\phi(x)=\left(\Phi_{0} / 2 \pi\right) \varphi(x)$ is the magnetic flux variable with $\Phi_{0}=h / 2 e$ the magnetic flux quantum and $\varphi(x)$ the phase of the macroscopic wave function describing the superconductor. The stationary modes are found by solving the eigenvalues and eigenvectors for the equation of motion (the Euler-Lagrange equations)

$$
\partial_{x}\left[l_{0}(x)^{-1} \partial_{x} \phi(x, t)\right]=c_{0}(x) \partial_{t}^{2} \phi(x, t),
$$

which is nothing but the wave equation in one dimension. The solution to this equation is expanded in normal modes and time-dependent amplitudes,

$$
\phi(x, t)=\sum_{n} q_{n}(t) u_{n}(x),
$$

such that $\ddot{q}_{n}=-\omega_{n} q_{n}$, with $\omega_{n}$ the resonator frequencies. Therefore the eigenstates $u_{n}$ satisfy the differential equation $\partial_{x}\left[l_{0}(x)^{-1} \partial_{x} u_{n}(x)\right]=-\omega_{n} c_{0}(x) u_{n}(x)$. The $u_{n}$ satisfy the orthogonal relation

$$
\int_{-l}^{l} c_{0, j}(x) u_{m, j}(x) u_{n, j}(x) d x=C_{r} \delta_{n m},
$$

with $C_{r}=\int_{-l}^{l} c_{0}(x) d x$ the total capacitance of the resonator.

For homogeneous resonators, $l_{0}$ and $c_{0}$ are constant, and we obtain the well-known case of equispaced eigenfrequencies $v_{n}=(2 n-1) / 2 l \sqrt{l_{0} c_{0}}$ with $2 l$ the length of the superconducting resonator and $u_{n}=\sqrt{2} \sin [(2 n-1) \pi x / 2 l]$. 


\section{Two coupled oscillators}

Consider the lumped element model depicted in Fig. 1(b). The discrete modes of the electromagnetic field inside the strips are described as arrays of $L C$ oscillators, together with the mutual inductances and capacitances, representing the cross-talk. By applying the current conservation law at each node of the circuit, we obtain a set of dynamical equations for the node fluxes $\phi_{j, n}$ :

$$
\begin{aligned}
& \Delta x c_{0}(n) \ddot{\phi}_{1, n}+\Delta x c_{m}(n)\left(\ddot{\phi}_{1, n}-\ddot{\phi}_{2, n}\right) \\
& =\frac{l_{0}(n)}{\Delta x} \frac{\left(\phi_{1, n-1}-\phi_{1, n}\right)}{l_{0}(n)^{2}-l_{m}(n)^{2}}-\frac{l_{0}(n)}{\Delta x} \frac{\left(\phi_{1, n}-\phi_{1, n+1}\right)}{l_{0}(n)^{2}-l_{m}(n+1)^{2}} \\
& \quad-\frac{l_{m}(n)}{\Delta x} \frac{\left(\phi_{2, n-1}-\phi_{2, n}\right)}{l_{0}(n)^{2}-l_{m}(n)^{2}}-\frac{l_{m}(n+1)}{\Delta x} \frac{\left(\phi_{2, n+1}-\phi_{2, n}\right)}{l_{0}(n)^{2}-l_{m}(n+1)^{2}},
\end{aligned}
$$

and the equivalent equation for the second resonator. Above, $c_{0}(n)$ and $l_{0}(n)$ are the capacitance and inductance per unit length, respectively, while $c_{m}(n)$ and $l_{m}(n)$ represent the mutual capacitance and mutual inductance coefficients. Notice that, in general, all these parameters are position dependent.

The former equations of motion are nothing but the EulerLagrange equations associated with the following Lagrangian:

$$
L=T-V
$$

with

$$
\begin{aligned}
T=\frac{\Delta x}{2} \sum_{n, j} c_{0} \dot{\phi}_{j, n}^{2}+c_{m}(n)\left(\dot{\phi}_{j, n}-\dot{\phi}_{j+1, n}\right)^{2}, \\
V=\frac{1}{2 \Delta x} \sum_{n, j} \frac{l_{0}}{l_{0}^{2}-l_{m}(n)^{2}}\left(\phi_{j, n}-\phi_{j, n-1}\right)^{2}-\frac{l_{m}(n)}{l_{0}^{2}-l_{m}(n)^{2}} \\
\times\left(\phi_{j, n}-\phi_{j, n-1}\right)\left(\phi_{j+1, n}-\phi_{j+1, n-1}\right) .
\end{aligned}
$$

We can now take the continuum limit $\Delta x \rightarrow 0$, which implies

(a) $\phi_{j, n} \rightarrow \phi_{j}(x)$,

(b) $\frac{\left(\phi_{j, n}-\phi_{j, n-1}\right)}{\Delta x} \rightarrow \partial_{x} \phi_{j}(x)$

(c) $\Delta x \sum_{n} \rightarrow \int_{-l}^{l} d x$.

Hence, the Lagrangian (A6) ends up as Eq. (1) in the main text that we rewrite here for completeness,

$$
\mathcal{L}=\frac{1}{2} \sum_{j=1,2} \int_{-l}^{l} d x\left[\hat{c}_{i j} \dot{\phi}_{i}(x) \dot{\phi}_{j}(x)-\hat{l}_{i j}^{-1} \partial_{x} \phi_{i}(x) \partial_{x} \phi_{j}(x)\right] .
$$

The fluxes $\phi_{i}(x)$ are thus coupled by the capacitance $\hat{c}(x)$ and inductance $\hat{l}(x)$ matrices given in the main text (2). The diagonal terms of these matrices represent the singleresonator Lagrangian $\mathcal{L}_{0}$ derived in the previous section, which depends on $l_{0}(x)$ and $c_{0}(x)$. On the other hand, the offdiagonal contributions represent the coupling Lagrangian $\mathcal{L}_{1}$, described by the parameters $l_{j j}=l_{0}, l_{i j}=l_{m}, c_{j j}=c_{0}+c_{m}$, and $c_{i j}=-c_{m}$.

\section{Generalization to more oscillators}

We now show that the quantum description of two coupled resonators presented above can be generalized to the case of $N$ coupled resonators. We therefore extend the sum in Eq. (A9) to $N$ :

$$
\mathcal{L}=\frac{1}{2} \sum_{i, j=1}^{N} \int_{-l}^{l} d x\left[\hat{c}_{i j} \dot{\phi}_{i}(x) \dot{\phi}_{j}(x)-\hat{l}_{i j}^{-1} \partial_{x} \phi_{i}(x) \partial_{x} \phi_{j}(x)\right],
$$

where the $\hat{c}(x)$ and $\hat{l}(x)$ are now given by $N \times N$ matrices with self-capacities (self-inductances) on the diagonal and the mutual capacities (self inductances) on the off diagonal. Following the same procedure as above we restrict ourselves to the fundamental modes, split off the single-resonator Lagrangians, and write the interaction part as

$$
\begin{aligned}
L_{1}= & \frac{1}{2} \sum_{N}\left(c_{m} \Delta_{1} \dot{q}_{j}^{2}-\frac{l_{m}^{2}}{l_{0}\left(l_{0}^{2}-l_{m}^{2}\right)} \Delta_{2} q_{j}^{2}\right) \\
& +\sum_{i=1}^{N-1}\left(-c_{m} \Delta_{1} \dot{q}_{i} \dot{q}_{i+1}+\frac{l_{m}}{l_{0}^{2}-l_{m}^{2}} \Delta_{2} q_{i} q_{i+1}\right),
\end{aligned}
$$

taking into account only nearest-neighbor interaction. The Hamiltonian can finally be written as

$$
\begin{aligned}
H / \hbar= & \sum_{j=1}^{N} \omega a_{j}^{\dagger} a_{j}-\sum_{j=1}^{N-1} g_{c}\left(a_{j}^{\dagger}-a_{j}\right)\left(a_{j+1}^{\dagger}-a_{j+1}\right) \\
& -\sum_{i=1}^{N-1} g_{i}\left(a_{j}^{\dagger}+a_{j}\right)\left(a_{j+1}^{\dagger}+a_{j+1}\right),
\end{aligned}
$$

with $\omega, g_{c}$, and $g_{i}$ the same as in the two-resonator case. In particular, from the resulting total Hamiltonian $H$ the normal frequencies can be found, giving

$$
\begin{aligned}
& \omega_{-}=\omega_{0} \sqrt{\frac{1}{1+2 C}\left(1+\frac{L}{v(1-L)}\right)}, \\
& \omega_{+}=\omega_{0} \sqrt{1-\frac{L}{v(1+L)}} .
\end{aligned}
$$

We finally notice that by making $l_{c} \rightarrow l(v \rightarrow 1)$, i.e., two straight parallel resonators, the formulas for the normal modes match the case of two coupled $L C$ circuits.

\section{APPENDIX B: LINEAR AND NONLINEAR COUPLINGS}

The motivation in this Appendix is twofold. On the one hand we estimate the validity of the linear approximation; on the other hand, we explicitly compute the first nonlinear corrections to the coupling.

We first expand the cosine in $\mathcal{L}_{\text {SQUID }}(11)$,

$$
\begin{aligned}
& -\cos \left(\frac{2 \pi}{\Phi_{0}} \phi_{-}\right) \\
& =-1+\frac{1}{2}\left(\frac{2 \pi}{\Phi_{0}}\right)^{2} \phi_{-}^{2}-\frac{1}{24}\left(\frac{2 \pi}{\Phi_{0}}\right)^{4} \phi_{-}^{4}+\cdots .
\end{aligned}
$$

We recall that

$$
\phi_{-}=u_{0} \sqrt{\hbar Z}\left(a_{-}^{\dagger}+a_{-}\right) \quad \text { and } \quad a_{-}=\frac{1}{\sqrt{2}}\left(a_{1}-a_{2}\right) \text {. }
$$


Assuming $Z=50 \Omega$ and defining

$$
\xi \equiv u_{0}^{2}\left(\frac{2 \pi}{\Phi_{0}}\right)^{2} 2 \hbar Z=u_{0}^{2} \frac{\pi^{2}}{10^{2}} \cong 10^{-1} u_{0}^{2},
$$

we can write for the expansion

$$
-\cos \left(\frac{2 \pi}{\Phi_{0}} \phi_{-}\right)=-1+\frac{\xi}{4}\left(a_{-}^{\dagger}+a_{-}\right)^{2}-\frac{\xi^{2}}{96}\left(a_{-}^{\dagger}+a_{-}\right)^{4} \text {. }
$$

\section{Linear regime}

The linear approximation is justified when the second-order terms in Eq. (B1), or equivalently the average value and fluctuations of the flux in Eq. (B4), are small. How does this relate to actual experiments? In order to determine a condition based on the number of photons we study the fluctuations $\left\langle\phi_{-}^{2}\right\rangle$, which are related to the expectation value

$$
\left\langle\left(a_{-}^{\dagger}+a_{-}\right)^{2}\right\rangle \cong 2\left(\left\langle a_{1}^{\dagger} a_{1}\right\rangle+\left\langle a_{2}^{\dagger} a_{2}\right\rangle\right) \equiv 4 n
$$

with $n$ the number of photons. Using the previous series we conclude that linearization is strictly justified whenever $n \ll$ $10 / u_{0}^{2}$, where $u_{0}$ is the value of the mode wave function. For the ring coupler layout, the same reasoning follows by replacing $u_{0} \rightarrow \partial_{x} u_{0} \Delta x$ in Eqs. (B2) and (B3).

\section{Nonlinear hopping terms}

With the help of Pathak's results, ${ }^{73}$ we compute,

$$
\begin{aligned}
\left(a_{-}+a_{-}^{\dagger}\right)^{4}= & a_{-}^{4}+4\left(a_{-}^{\dagger}\right)^{3} a_{-}+6\left(a_{-}^{\dagger}\right)^{2} a_{-}^{2} \\
& +6 a^{\dagger} a+3+\text { H.c. }
\end{aligned}
$$

Notice that $a_{-}=\frac{1}{\sqrt{2}}\left(a_{1}-a_{2}\right)$ [Eq. (B2)], so the above ends up in a long expression hard to deal with. To make it simpler, we assume that sidebands will select $a_{-}^{2}$ or $a^{\dagger} a$ and we resort to a RWA argument to write

$$
\left(a_{-}+a_{-}^{\dagger}\right)^{4} \cong 6\left(a_{-}^{\dagger}\right)^{2} a_{-}^{2}+12 a^{\dagger} a+6\left(a_{-}^{\dagger}\right)^{2}+6 a_{-}^{2}+3 .
$$

Looking at each term we rewrite them in terms of the local bosonic operators $a_{1}$ and $a_{2}$ :

$$
\begin{aligned}
4\left(a_{-}^{\dagger}\right)^{2} a_{-}^{2}= & \left(a_{1}^{\dagger}\right)^{2} a_{1}^{2}+\left(a_{2}^{\dagger}\right)^{2} a_{2}^{2}+2 a_{1}^{\dagger} a_{2}^{\dagger} a_{1} a_{2} \\
& -2 a_{1}^{\dagger} a_{2}^{\dagger}\left(a_{1}^{2}+a_{2}^{2}\right)+\text { H.c. },
\end{aligned}
$$

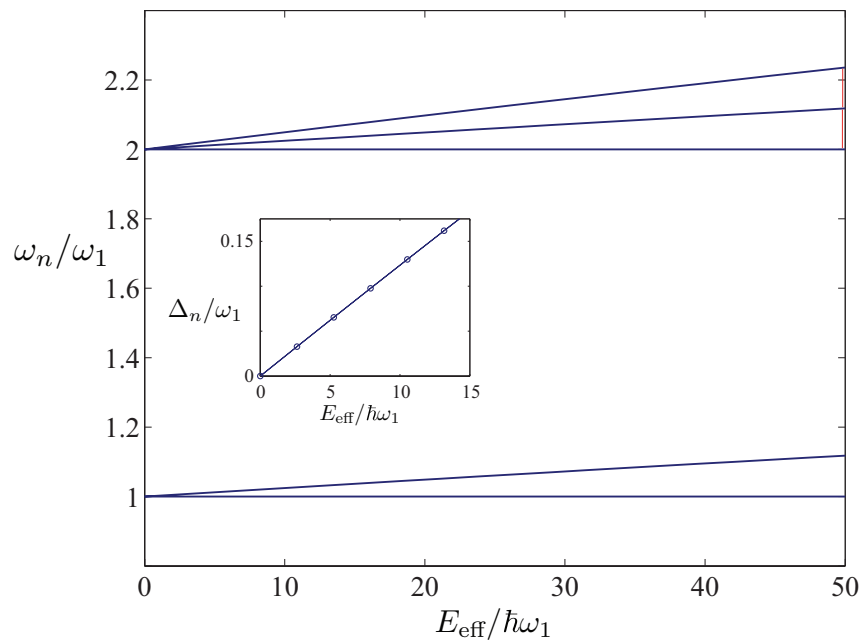

FIG. 5. (Color online) Energies of the full Hamiltonian of two $L C$ resonators with frequency $\omega_{1}$ coupled by a SQUID (11), as a function of the effective Josephson energy $E_{\text {eff }}$. We plot the transition frequencies relative to the ground state, $\omega_{n}=\left(E_{n}-E_{0}\right) / \hbar$. Note the splitting between energy levels which is linear with $E_{\text {eff }}$. The inset shows the energy splitting $\Delta_{n}$, for $n=1$ (solid) and 2 (circles) photons, in perfect agreement with our linearized theory.

$$
\begin{gathered}
2\left(a_{-}^{\dagger}\right)^{2}=\left(a_{1}^{\dagger}\right)^{2}+\left(a_{2}^{\dagger}\right)^{2}-2 a_{1}^{\dagger} a_{2}^{\dagger}, \\
2 a_{-}^{\dagger} a_{-}=a_{1}^{\dagger} a_{1}+a_{2}^{\dagger} a_{2}-a_{1}^{\dagger} a_{2}-a_{2}^{\dagger} a_{1} .
\end{gathered}
$$

\section{Full diagonalization}

We conclude this Appendix by computing the full diagonalization of the Hamiltonian associated with Eq. (11). More precisely, we consider two zero-mode resonators connected by a single SQUID element and diagonalize everything in the Fock basis of the oscillators. The SQUID acts as a nonlinearity whose bounded strength can be faithfully reproduced using Fock operators in a truncated basis. In Fig. 5 we show the resulting spectrum, with the energy splitting induced by the effective coupling, which is approximately linear in the SQUID strength. Note that for a small number of photons the energy splitting is the same, demonstrating the linear behavior of the element.
${ }^{1}$ A. Blais, R.-S. Huang, A. Wallraff, S. M. Girvin, and R. J. Schoelkopf, Phys. Rev. A 69, 062320 (2004).

${ }^{2}$ A. Wallraff, D. Schuster, A. Blais, L. Frunzio, R.-S. Huang, J. Majer, S. Kumar, S. M. Girvin, and R. J. Schoelkopf, Nature (London) 431, 162 (2004).

${ }^{3}$ T. Niemczyk, F. Deppe, H. Huebl, E. P. Menzel, F. Hocke, M. J. Schwarz, J. J. Garcia-Ripoll, D. Zueco, T. Hümmer, E. Solano et al., Nat. Phys. 6, 772 (2010).

${ }^{4}$ P. Forn-Díaz, J. Lisenfeld, D. Marcos, J. J. García-Ripoll, E. Solano, C. J. P. M. Harmans, and J. E. Mooij, Phys. Rev. Lett. 105, 237001 (2010).

${ }^{5}$ T. Hime, P. A. Reichardt, B. L. T. Plourde, T. L. Robertson, C.-E. Wu, A. V. Ustinov, and J. Clarke, Science 314, 1427 (2006).
${ }^{6}$ A. O. Niskanen, K. Harrabi, F. Yoshihara, Y. Nakamura, S. Lloyd, and J. S. Tsai, Science 316, 723 (2007).

${ }^{7}$ S. H. W. van der Ploeg, A. Izmalkov, A. M. van den Brink, U. Hübner, M. Grajcar, E. Il'ichev, H.-G. Meyer, and A. Zagoskin, Phys. Rev. Lett. 98, 057004 (2007).

${ }^{8}$ O. Astafiev, A. M. Zagoskin, A. A. Abdumalikov, Y. A. Pashkin, T. Yamamoto, K. Inomata, Y. Nakamura, and J. S. Tsai, Science 327, 840 (2010).

${ }^{9}$ A. A. Abdumalikov, O. Astafiev, A. M. Zagoskin, Y. A. Pashkin, Y. Nakamura, and J. S. Tsai, Phys. Rev. Lett. 104, 193601 (2010).

${ }^{10}$ C. Sabín, M. del Rey, J. J. García-Ripoll, and J. León, Phys. Rev. Lett. 107, 150402 (2011). 
${ }^{11}$ A. L. Rakhmanov, A. M. Zagoskin, S. Savel'ev, and F. Nori, Phys. Rev. B 77, 144507 (2008).

${ }^{12}$ C. Hutter, E. A. Tholén, K. Stannigel, J. Lidmar, and D. B. Haviland, Phys. Rev. B 83, 014511 (2011).

${ }^{13}$ D. Zueco, J. J. Mazo, E. Solano, and J. J. García-Ripoll, Phys. Rev. B 86, 024503 (2012).

${ }^{14}$ A. M. Zagoskin, A. L. Rakhmanov, S. Savel'ev, and F. Nori, Phys. Status Solidi B 246, 955 (2009).

${ }^{15}$ P. D. Nation, M. P. Blencowe, A. J. Rimberg, and E. Buks, Phys. Rev. Lett. 103, 087004 (2009).

${ }^{16}$ G. Romero, J. J. García-Ripoll, and E. Solano, Phys. Rev. Lett. 102, 173602 (2009).

${ }^{17}$ Y.-F. Chen, D. Hover, S. Sendelbach, L. Maurer, S. T. Merkel, E. J. Pritchett, F. K. Wilhelm, and R. McDermott, Phys. Rev. Lett. 107, 217401 (2011).

${ }^{18}$ B. Peropadre, G. Romero, G. Johansson, C. M. Wilson, E. Solano, and J. J. García-Ripoll, Phys. Rev. A 84, 063834 (2011).

${ }^{19}$ I.-C. Hoi, C. M. Wilson, G. Johansson, T. Palomaki, B. Peropadre, and P. Delsing, Phys. Rev. Lett. 107, 073601 (2011).

${ }^{20}$ M. J. Hartmann, F. G. S. L. Brandão, and M. B. Plenio, Nat. Phys. 2, 849 (2006).

${ }^{21}$ D. G. Angelakis, M. F. Santos, and S. Bose, Phys. Rev. A 76, 031805 (2007).

${ }^{22}$ M. Leib and M. J. Hartmann, New J. Phys. 12, 093031 (2010).

${ }^{23}$ M. Leib, F. Deppe, A. Marx, R. Gross, and M. Hartmann, New J. Phys. 14, 075024 (2012).

${ }^{24}$ I. Carusotto, D. Gerace, H. E. Tureci, S. De Liberato, C. Ciuti, and A. Imamoğlu, Phys. Rev. Lett. 103, 033601 (2009).

${ }^{25}$ A. Nunnenkamp, J. Koch, and S. M. Girvin, New J. Phys. 13, 095008 (2011).

${ }^{26}$ J. R. Johansson, G. Johansson, C. M. Wilson, and F. Nori, Phys. Rev. Lett. 103, 147003 (2009).

${ }^{27}$ C. M. Wilson, G. Johansson, A. Pourkabirian, J. R. Johansson, T. Duty, F. Nori, and P. Delsing, Nature (London) 479, 376 (2011).

${ }^{28}$ M. Mariantoni, F. Deppe, A. Marx, R. Gross, F. Wilhelm, and E. Solano, Phys. Rev. B 78, 104508 (2008).

${ }^{29}$ G. M. Reuther, D. Zueco, F. Deppe, E. Hoffmann, E. P. Menzel, T. Weißl, M. Mariantoni, S. Kohler, A. Marx, E. Solano, R. Gross, and P. Hänggi, Phys. Rev. B 81, 144510 (2010).

${ }^{30}$ D. I. Tsomokos, S. Ashhab, and F. Nori, Phys. Rev. A 82, 052311 (2010).

${ }^{31}$ L. Tian, M. S. Allman, and R. W. Simmonds, New J. Phys. 10, 115001 (2008).

${ }^{32}$ J. Bourassa, J. M. Gambetta, A. A. Abdumalikov, O. Astafiev, Y. Nakamura, and A. Blais, Phys. Rev. A 80, 032109 (2009).

${ }^{33}$ J.-Q. Liao, Z. R. Gong, L. Zhou, Y.-x. Liu, C. P. Sun, and F. Nori, Phys. Rev. A 81, 042304 (2010).

${ }^{34}$ E. Zakka-Bajjani, F. Nguyen, M. Lee, L. R. Vale, R. W. Simmonds, and J. Aumentado, Nat. Phys. 7, 599 (2011).

${ }^{35}$ E. Flurin, N. Roch, F. Mallet, M. H. Devoret, and B. Huard, Phys. Rev. Lett. 109, 183901 (2012).

${ }^{36}$ F. Nguyen, E. Zakka-Bajjani, R. W. Simmonds, and J. Aumentado, Phys. Rev. Lett. 108, 163602 (2012).

${ }^{37}$ L. Chirolli, G. Burkard, S. Kumar, and D. P. DiVincenzo, Phys. Rev. Lett. 104, 230502 (2010).

${ }^{38}$ J. Koch, A. A. Houck, K. L. Hur, and S. M. Girvin, Phys. Rev. A 82, 043811 (2010).

${ }^{39}$ H. Brenning, S. Kubatkin, and P. Delsing, J. Appl. Phys. 96, 6822 (2004).
${ }^{40}$ N. C. Menicucci, P. van Loock, M. Gu, C. Weedbrook, T. C. Ralph, and M. A. Nielsen, Phys. Rev. Lett. 97, 110501 (2006).

${ }^{41}$ M. Hofheinz, H. Wang, M. Ansmann, R. C. Bialczak, E. Lucero, M. Neeley, A. D. O'Connell, D. Sank, J. Wenner, J. M. Martinis et al., Nature (London) 459, 546 (2009).

${ }^{42}$ W. E Shanks, D. L. Underwood, and A. A. Houck, arXiv:1303.0874.

${ }^{43}$ J. R. Johansson, G. Johansson, C. M. Wilson, and F. Nori, Phys. Rev. A 82, 052509 (2010).

${ }^{44}$ C. R. Paul, Analysis of Multiconductor Transmission Lines (WileyInterscience, New York, 1994).

${ }^{45}$ C. R. Paul, IEEE Trans. Electron. Compat. 44, 413 (2002).

${ }^{46}$ M. Sandberg, C. M. Wilson, F. Persson, T. Bauch, G. Johansson, V. Shumeiko, T. Duty, and P. Delsing, Appl. Phys. Lett. 92, 203501 (2008).

${ }^{47}$ M. A. Castellanos-Beltran and K. W. Lehnert, Appl. Phys. Lett. 91, 083509 (2007).

${ }^{48}$ M. A. Castellanos-Beltran, K. D. Irwin, G. C. Hilton, L. R. Vale, and K. W. Lehnert, Nat.. Phys. 4, 929 (2008).

${ }^{49}$ T. Yamamoto, K. Inomata, M. Watanabe, K. Matsuba, T. Miyazaki, W. D. Oliver, Y. Nakamura, and J. S. Tsai, Appl. Phys. Lett. 93, 042510 (2008).

${ }^{50}$ M. Grajcar, Y.-x. Liu, F. Nori, and A. M. Zagoskin, Phys. Rev. B 74, 172505 (2006).

${ }^{51}$ M. H. Devoret, in Quantum Fluctuations: Les Houches Session LXIII, edited by S. Reynaud, E. Giacobino, and J. Zinn-Justin (Elsevier, North-Holland, Amsterdam, 1997), pp. 351-386.

${ }^{52}$ J. Clarke, Proc. IEEE 77, 1208 (1989).

${ }^{53}$ B. Peropadre, P. Forn-Díaz, E. Solano, and J. J. García-Ripoll, Phys. Rev. Lett. 105, 023601 (2010).

${ }^{54}$ M. J. Everitt, T. D. Clark, P. Stiffell, H. Prance, R. J. Prance, A. Vourdas, and J. F. Ralph, Phys. Rev. B 64, 184517 (2001).

${ }^{55}$ M. J. Everitt, P. Stiffell, T. D. Clark, A. Vourdas, J. F. Ralph, H. Prance, and R. J. Prance, Phys. Rev. B 63, 144530 (2001).

${ }^{56}$ M. J. Everitt, T. D. Clark, P. B. Stiffell, A. Vourdas, J. F. Ralph, R. J. Prance, and H. Prance, Phys. Rev. A 69, 043804 (2004).

${ }^{57}$ Typically this happens at the middle point for the fundamental mode. This is equivalent to choosing the phase $\varphi_{1}\left(x_{1}\right)=$ $2 \pi \phi_{1}\left(x_{1}\right) / \Phi_{0}=\varphi_{2}\left(x_{2}\right)=2 \pi \phi_{2}\left(x_{2}\right) / \Phi_{0}=0$ and neglecting the phase drop along the connecting wires compared to that across the Josephson junction. In this case the fluxoid quantization in the loop reads $\Delta \phi_{1}-\Delta \phi_{2}+\phi_{J}+\Phi_{\circlearrowright}=n \Phi_{0}$. The former assumption can always be made since the current flowing in the resonator depends only on the spatial derivative of the phase and not on its absolute value. The latter is a good approximation as long as the Josephson inductance is large compared to the kinetic inductance of the wires. This is the case as long as the superconducting wire is not made extremely narrow.

${ }^{58}$ T. P. Orlando and K. A. Delin, Foundations of Applied Superconductivity (Addison-Wesley, New York, 1991).

${ }^{59}$ J. Bourassa, F. Beaudoin, J. M. Gambetta, and A. Blais, Phys. Rev. A 86, 013814 (2012).

${ }^{60}$ M. Schiró, M. Bordyuh, B. Öztop, and H. E. Türeci, Phys. Rev. Lett. 109, 053601 (2012).

${ }^{61}$ C. M. Wilson and P. Forn-Díaz (private communication).

${ }^{62}$ M. Eckholt and J. J. García-Ripoll, Phys. Rev. A 77, 063603 (2008).

${ }^{63}$ F. Galve, L. A. Pachón, and D. Zueco, Phys. Rev. Lett. 105, 180501 (2010).

${ }^{64}$ M. Cramer and J. Eisert, New J. Phys. 8, 71 (2006).

${ }^{65}$ J. Eisert and D. Gross, Phys. Rev. Lett. 102, 240501 (2009). 
${ }^{66}$ M. Cramer, A. Serafini, and J. Eisert, in Quantum Information and Many Body Quantum Systems, edited by S. M. M. Ericsson, CRM Series No. 8 (Publications of the Scuola Normale Superiore, Pisa, 2008), pp. 51-72.

${ }^{67}$ M. B. Plenio, J. Hartley, and J. Eisert, New J. Phys. 6, 36 (2004).

${ }^{68}$ J. J. García-Ripoll, New J. Phys. 8, 305 (2006).

${ }^{69}$ Note that we mention DMRG because of its widespread use in the field of Bose-Hubbard simulations (Ref. 70) and its enormous potential for nonequilibrium and dissipative dynamics (Ref. 71). In some context, such as weak nonlinearities, other methods based on the Heisenberg picture or stochastic equations could be used.

${ }^{70}$ A. J. Daley, C. Kollath, U. Schollwck, and G. Vidals, J. Stat. Mech. (2004) P04005.

${ }^{71}$ J. Prior, A. W. Chin, S. F. Huelga, and M. B. Plenio, Phys. Rev. Lett. 105, 050404 (2010).

${ }^{72}$ J. Johansson, P. Nation, and F. Nori, Comput. Phys. Commun. 184, 1234 (2013).

${ }^{73}$ A. Pathak, J. Phys. A 33, 5607 (2000). 